\title{
Pós-alta em Hanseníase no Ceará: limitação da atividade funcional, consciência de risco e participação social
}

\author{
Post-treatment of Leprosy in Ceará: activity and functional limitation, safety awareness and social participation
}

Pos-alta hospitalária en Lepra en Ceará: limitación de actividad funcional, conciencia de riesgo y participación social

\author{
JaQueline Caracas Barbosa', Alberto Novaes Ramos I $\mathbf{r}^{1}$, \\ Maria de Jesus Freitas Alencar" Cláudio Gastão JunQueira de Castro"II \\ 'Universidade Federal do Ceará. Faculdade de Medicina, Departamento de Saúde Comunitária. Fortaleza, CE \\ "Secretaria Estadual de Saúde de Rondônia. Programa de Controle de Hanseníase e Tuberculose. Porto Velho, RO \\ "I'Universidade de São Paulo. Faculdade de Saúde Pública, Departamento de Práticas. São Paulo, SP
}

Submissão: $28 / 08 / 2008$

Aprovação: 10/10/2008

\section{RESUMO}

Caracterizar a limitação funcional, de atividade, consciência de risco, e restrição à participação social em pessoas atingidas pela hanseníase no pós-alta. Estudo seccional-descritivo com 69 residentes em Sobral, Ceará, com alta entre 2003 a 2005 . Foram realizados exame físico dermato-neurológico, avaliação demográfica, de limitação funcional-atividade-consciência de risco e de restrição à participação social. Vinte $(28,9 \%)$ apresentaram escores SALSA 19 e 20 e escore EHF zero. O maior escore EHF foi alcançado por dois participantes, com 25 e 28 na escala SALSA. Na escala de participação 37 (53,6\%) não apresentaram restrição e tinham escore EHF zero. Dois (2,9\%) com escore EHF zero tinham leve restrição e 1 ( $1,5 \%)$, grande restrição. Reafirma-se a potencialidade destas ferramentas para a atenção integral aos portadores.

Descritores: Hanseníase/reabilitação; Risco; Atividades cotidianas; Participação social.

\section{ABSTRACT}

To characterize the functional limitation, activity limitation, risk conscience, and the social participation in people reached by hansen's disease in the post-MDT period. Cross-sectional, descriptive study, accomplished in 2006. Sixty-nine residents in Sobral that had discharge from MDT between 2003-2005 participated. The subjects were interviewed: demographic evaluation, dermato-neurological exams, evaluation of functional limitation-activity-risk conscience and the restriction in social participation. Twenty (28.9\%) presented SALSA scores 19 and 20 and EHF score zero. The largest EHF score was reached by two participants, with scores 25 and 28 in the SALSA scale. In the participation scale 37 (53.6\%) didn't present restriction and had EHF scores zero. Two (2.9\%) with EHF score zero had mild restriction, and 1 (1.5\%) severe restriction. This study reaffirms the potentiality of these tools for integral care of people reached.

Descriptors: Leprosy/rehabilitation; Risk; Activities of daily living; Consumer Participation.

\section{RESUMEN}

Caracterizar la limitación funcional de actividad, conciencia de riesgo y restricción a la participación social en personas atingidas por la lepra en el post-alta. Estudio seccional y descriptivo con 69 sitiados en Sobral, Ceará, con alta entre 2003 y 2005 . Fueron hechos examen físico dermatológico y además neurológico, evaluación demográfica, de limitación funcional, actividad y conciencia de riesgo y de restricción a la participación social. Veinte (28,9\%) presentaron escores SALSA 19 y 20 escore EHF cero. La mayor EHF fue alcanzada por dos participantes, con 25 y 28 años en la escala SALSA. En la categoría participación, $37(53,6 \%)$ no presentaron restricción y tenían escore EHF cero. Dos $(2,9 \%)$ con EHF cero tenían poca restricción y 1 (1,5\%), gran restricción. Delante de eso, se afirma una vez más la potencialidad de éstas herramientas para la atención integral a los portadores.

Descriptores: Lepra/rehabilitación; Riesgo; Actividades cotidianas; Participación comunitaria.

Pesquisa financiada com recursos provenientes do Centro Nacional de Desenvolvimento Científico e Tecnológico/CNPQ através do edital MCT-CNPQ/MS DAB/ SAS n. 49/2005.

Correspondência: Jaqueline Caracas Barbosa. Rua Prof. Costa Mendes, I608. $5^{\circ}$ andar, Bairro Rodolfo Teófilo. CEP 60430-I40. Fortaleza, CE. 


\section{INTRODUÇÃO}

A hanseníase representa um processo infeccioso crônico de elevada magnitude. Em todo o mundo, 254.525 casos novos foram detectados no ano de 2007. O Brasil contribuiu com 15,4\% (39.125) desses casos, sendo o país com maior número de casos no continente Americano (93,2\% - 39. 125 entre 41.978 casos do continente) $)^{(1)}$.

De forma adicional, nesse mesmo ano o país mantém um coeficiente médio de detecção em menores de Quinze anos de idade considerado como muito alto, de 0,66 casos novos por 10.000 habitantes ${ }^{(2,3)}$. Nos últimos cinco anos, a média de casos novos detectados no Brasil nessa população é de 4.000 casos indicando um processo de transmissão recente e ativo da doença $a^{(3)}$.

A especificidade do Mycobacterium leprae pelo acometimento de nervos periféricos gera alterações motoras e sensoriais potenciais com impactos significativos do ponto de vista físico, social e econômico $^{(4-6)}$.

Considerando-se a natureza incapacitante, a baixa gravidade e o estigma relacionado ${ }^{(7)}$ um grande contingente de pessoas Que vivem com hanseníase apresenta necessidades marcantes com vistas à reabilitação ao longo de suas vidas ${ }^{(8-10)}$. Para a abordagem mais integral das pessoas, o período após a conclusão da polieuimioterapia (PQT), reconhecido como momento do "pós-alta", também deve ser considerado como relevante, além do momento do diagnóstico e do período de tratamento com a PQT, em uma perspectiva de longitudinalidade do cuidado e da integralidade ${ }^{(1)}$.

Nessa abordagem, Questões como deficiência, limitação de atividade e restrição de participação social são fundamentais e devem ser necessariamente consideradas. Essas dimensões foram definidas na Classificação Internacional de Funcionalidade, Incapacidade e Saúde como componentes significativos do estado de saúde de uma pessoa $^{(12)}$.

A necessidade de determinar a dimensão e a natureza desses problemas e de monitorar a avaliação de prevenção de deficiências e incapacidades e reabilitação torna-se cada vez mais necessária, mesmo em cenários de alcance do controle ${ }^{(6,12)}$. Diversas propostas vêm sendo desenvolvidas. Mais recentemente, duas escalas foram desenvolvidas com vistas à avaliação da limitação de atividade e da consciência de risco (escala SALSA - Screening of Activity Limitation and Safety Awarenes) ${ }^{9}$ e da restrição à participação social ${ }^{(6,13)}$. Além de validadas, ambas as escalas estão sendo aplicadas em diferentes cenários sócio-culturais.

O objetivo do presente estudo é caracterizar a limitação funcional, limitação de atividade, consciência de risco, e a restrição à participação social em pessoas atingidas pela hanseníase Que se encontram no momento do pós-alta no Município de Sobral, Ceará, Brasil.

\section{METODOLOGIA}

Estudo seccional, desenvolvido no Município de Sobral, Ceará, Nordeste do Brasil, realizado em 2006. A seleção justificou-se por: ser um dos sete municípios prioritários para as ações de controle da hanseníase ${ }^{(14)}$, representar um município com características rurais e com elevada cobertura populacional do Programa Saúde da Família no Estado do Ceará, a maior entre os municípios prioritários. O município de Sobral situa-se na região Norte do Ceará, a 240
Quilômetros da capital, Fortaleza. Em 2007 sua população estimada era de 175.814 habitantes $^{(15)}$.

A população do estudo consistiu de uma amostra de 10\% dos pacientes com hanseníase Que receberam alta por cura do tratamento de PQT para hanseníase no período de 2003 a 2005. A base populacional foi definida a partir do banco de dados do Sistema de Informação de Agravos de Notificação (SINAN / Ministério da Saúde), específico para a hanseníase. Essa amostra foi estratificada segundo classificação operacional (paucibacilar e multibacilar) e grau de incapacidade física (Graus 0, I e 2/3) no momento da alta. Os participantes residiam no Município de Sobral e tinham mais de 15 anos de idade. Um total de 69 indivíduos foi selecionado.

Os indivíduos foram entrevistados e avaliados clinicamente. Para a avaliação demográfica (sexo, idade e ocupação) foi aplicado instrumento padronizado.

Para dimensionar a limitação funcional, foi realizado exame físico dermato-neurológico padronizado e sistematizado ${ }^{(16)}$ para definição do escore Olho-Mão-Pé (Eye-Hand-Foot / EHF) ${ }^{(17)}$. Este escore dimensiona a gravidade do comprometimento da incapacidade física, por criar um somatório do grau de incapacidade segundo seguimentos (olho, mão e pé) e dimídios (esquerdo e direito), com valor máximo de 12. O escore EHF foi utilizado como uma alternativa ao grau máximo de incapacidade definido pela Organização Mundial da Saúde (OMS) para caracterização da incapacidade física ${ }^{(18)}$.

Para dimensionar a limitação de atividade e consciência de risco foi utilizada a Escala SALSA (Screening of Activity Limitation and Safety Awareness), desenvolvida para aplicação no diabetes mellitus, hanseníase ou outras neuropatias periféricas. O objetivo principal era realizar triagem da extensão da limitação de atividade e o risco de se aumentar as deficiências, com versão em português do Brasil já validada. Ela abrange Quatro domínios envolvendo mãos, pés e auto-cuidado e também gera um escore final, Que varia de I a 80 , mas sem pontos de corte para definir padrões. De uma forma geral, um escore baixo indica pouca dificuldade com atividades da vida diária, enquanto Que escores mais altos indicam níveis crescentes de limitação de atividade. Já o escore da consciência de risco é calculado separadamente do escore SALSA por meio do somatório das opções demarcadas com círculo nas colunas "não consigo fisicamente" com "evito por causa do risco". O escore de consciência de risco será um valor Que varia de 0 a 11 . Valores mais altos indicam uma consciência crescente dos riscos envolvidos em certas atividades, mas também indicam Que há uma limitação de atividade devido a esse fato ${ }^{(9,19)}$.

Para dimensionar a restrição a participação social foi utilizada a Escala de Participação. Essa escala foi elaborada para ser usada em países onde a hanseníase mantém-se com elevado padrão de endemicidade e também possui versão em português do Brasil já validada. É utilizada para triagem e dimensionamento de restrições (percebidas) à participação de pessoas afetadas por hanseníase, deficiências ou outras condições estigmatizantes ${ }^{(6,13)}$. Abrange oito das nove principais áreas da vida definidas na $\mathrm{CIF}^{(11)}$. Deve ser aplicada a pessoas com 15 anos de idade ou mais e é composta por 18 itens. O escore final da escala varia de 0 a 90 , sendo que o ponto de corte recomendado como não tendo restrição à participação é de 12 pontos. Acima desse valor, identificam-se diferentes graus de restrição: leve restrição (1 3 a 22), moderada restrição (23 a 32), grande restrição (33 a 52) e extrema restrição (53 a 90) $)^{(6,13)}$. 
O processo de coleta dos dados demográficos, de limitação da atividade/consciência de risco e de restrição à participação foi realizado por dois profissionais de saúde habilitados. A avaliação clínica funcional foi realizada por um único profissional de referência em hanseníase.

Toda a análise descritiva dos dados foi realizada por meio do software Epi-Info 6.04d. O estudo foi aprovado pelo Comitê de Ética em Pesquisa da Faculdade de Saúde Pública da Universidade de São Paulo (Número do Protocolo: 1512 ).

\section{RESULTADOS E DISCUSSÃO}

Dos 69 participantes do estudo $43(62,3 \%)$ eram do sexo feminino e $26(37,7 \%)$ do sexo masculino. A idade variou entre 18 e 78 com média de 46 anos e mediana de 45 anos. Cinqüenta e Quatro $(79,8 \%)$ pessoas tinham alguma ocupação, 9 (13,0\%) eram aposentados e apenas $5(7,2 \%)$ desempregados. A ocupação mais freeüente foi atividades do lar - $26(37,7 \%)$, seguida por agente comunitário de saúde - 4 (5,8\%); costureira, servente de pedreiro e estudante - 2 (2,9\%). Com relação à classificação operacional 9 $(13,0 \%)$ eram paucibacilares (PB) e $60(87,0 \%)$ multibacilares (MB).

A Tabela 1 apresenta os resultados do escore total EHF Que variou de 0 a 8 pontos.

O escore SALSA variou de 19 a 64 pontos. A pontuação com maior freqüência de indivíduos foi a de 20 pontos, observada em 16 $(23,2 \%)$ participantes, seguida por 9 (13\%) participantes Que pontuaram 19 pontos. Escores acima de 50 pontos foram alcançados somente por I $(1,4 \%)$ participante. O Gráfico I apresenta a distribuição do escore na população do estudo.

O escore de consciência de risco (escala de 0 a 11 ), nesse estudo variou de 0 a $7: 39(56,5 \%)$ pontuaram $0,17(24,6 \%)$ pontuaram I, 6 $(8,7 \%)$ pontuaram 2,1 I $(1,4 \%)$ pontuou $3,2(2,9 \%)$ pontuaram 4,1 $(1,4 \%)$ pontuou 5 e 2 (2,9\%) pontuaram 6 e l $(1,4 \%)$ pontuou 7.

Na correlação do escore EHF e escore SALSA constatou-se Que 20 $(28,9 \%)$ participantes apresentaram escores SALSA 19 e 20 e escore EHF zero. lá o maior escore EHF foi alcançado por dois participantes, um Que pontuou 25 e outro 28 na escala SALSA. A análise combinada dos escores SALSA e EHF é apresentada no Gráfico 2.

A classificação final da escala de participação mostrou Que 64 $(92,8 \%)$ pessoas não apresentaram nenhuma restrição significativa, $4(5,8 \%)$ leve restrição e somente $1(1,4 \%)$, grande restrição. Os demais itens moderada restrição e extrema restrição não foram pontuados.

Tabela 1. Escore (número absoluto e percentual) EHF da população do estudo.

\begin{tabular}{lcc}
\hline Escore EHF & Número & Percentual \\
\hline 0 & 41 & 59,4 \\
1 & 7 & 10,2 \\
2 & 5 & 7,2 \\
3 & 4 & 5,8 \\
4 & 7 & 10,2 \\
5 & 2 & 2,9 \\
7 & 1 & 1,4 \\
8 & 2 & 2,9 \\
\hline Total & 69 & 100 \\
\hline
\end{tabular}

Na correlação entre o escore SALSA e a classificação da escala de participação constatou-se Que a totalidade dos participantes classificada como sem restrição a participação social estava distribuída em praticamente todos os escores da escala SALSA. O participante Que foi classificado como tendo extrema restrição à participação social atingiu apenas 23 pontos no escore SALSA (Gráfico 3).

A maioria dos participantes ( 37 - 53,6\%) não apresentou restrição e tinha escore EHF de zero. Chama a atenção o fato de Que 2 (2,9\%) participantes com escore EHF de zero tinham leve restrição e I (1,5\%), grande restrição, conforme mostra o Gráfico 4.

O presente estudo tomou como base para a sua realização casos de hanseníase identificados a partir do SINAN, com identificação de endereços. Do total selecionado inicialmente houve necessidade de amostragem aleatória adicional em 40 casos (58\%) em virtude de situações como não localização (3 I pessoas por: mudança de município, não localização do endereço registrado, não resposta por contato no endereço inicialmente identificado), recusa de participação no estudo (2), relatos como "sem condições psicológicas" (1), idade menor do Que 15 anos (5) e óbito (1). Com a abordagem realizada manteve-se a proposta numérica da amostral original. Essa situação reflete o contexto operacional real da base de dados nacional e não representou prejuízo na análise, considerando-se o objetivo central do estudo.

Uma Questão a ser considerada é o fato de as escalas utilizadas neste estudo terem sido recentemente validadas em diferentes países (incluindo o Brasil) $)^{(6,9,13,19)}$ e estarem ainda em processo de utilização mais ampliado em outros cenários. Esta situação é refletida na imprecisão de algumas informações dentro do manual de instruções, o Que dá margem a desvios no processo originalmente definido para utilização. Ressalta-se também Que ambas as escalas ainda estão sendo utilizadas basicamente em atividades de pesQuisa e não na rotina dos serviços no Brasil. Para controlar essas Questões foram realizados contatos com as equipes Que desenvolveram ambas as escalas para esclarecimentos e sugestionamentos com vistas ao aperfeiçoamento.

Com relação à escala SALSA, ressalta-se o desafio da abordagem de múltiplas dimensões em uma única medida Que aborda dimensões Qualitativas em uma escala Quantitativa. No presente estudo foi considerado o aspecto de uma pessoa conseguir fazer ou não determinada atividade e não apenas se ela realizava a atividade ou não. lá para a escala de participação, considerou-se a sua aplicação de fácil execução. O manual é completo e o pressuposto de comparação com "pares" foi passível de trabalho junto às pessoas atingidas pela hanseníase. Entretanto, esse aspecto foi levantado como um dos pontos Questionáveis da escala e Que pode, potencialmente, dificultar a compreensão $0^{(20)}$.

A população do estudo era composta principalmente por mulheres, não refletindo os dados nacionais e do estado ${ }^{(2,3,21)}$ e da literatura ${ }^{(5)}$. Chama a atenção Que o processo amostral aleatório baseado na estratificação por forma clínica e grau de incapacidade contribui para essa análise. A caracterização etária da população do estudo traduz um contingente de pessoas Que está em sua vida produtiva, fato este refletido no fato de $79,8 \%$ terem alguma ocupação, no mais das vezes sem vínculo empregatício com vinculação ao mercado de trabalho informal, refletindo a base populacional da Qual advém.

Com relação à classificação operacional a maior freeüência $(87,0 \%)$ 


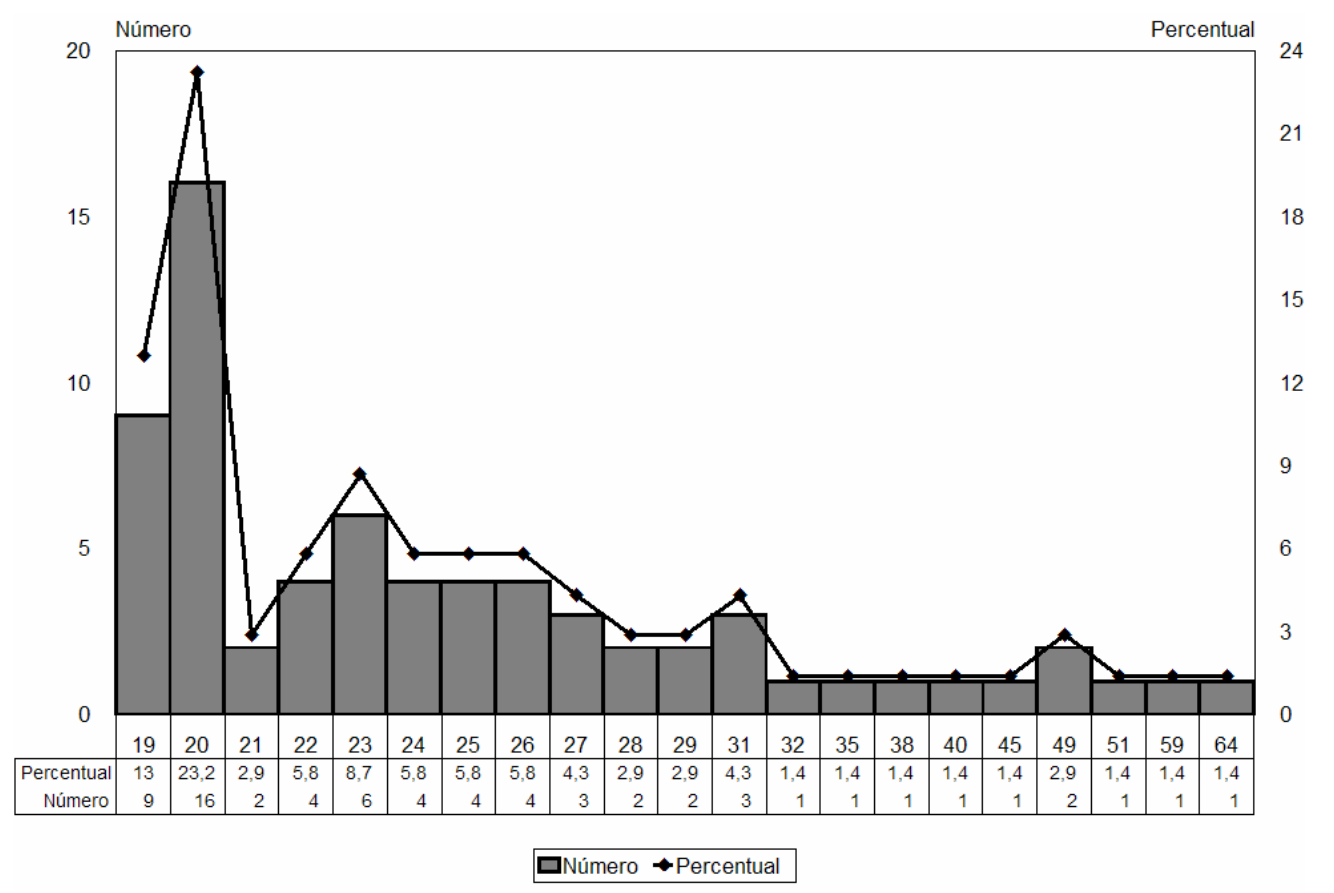

Gráfico I. Escore (número absoluto e percentual) SALSA da população do estudo.

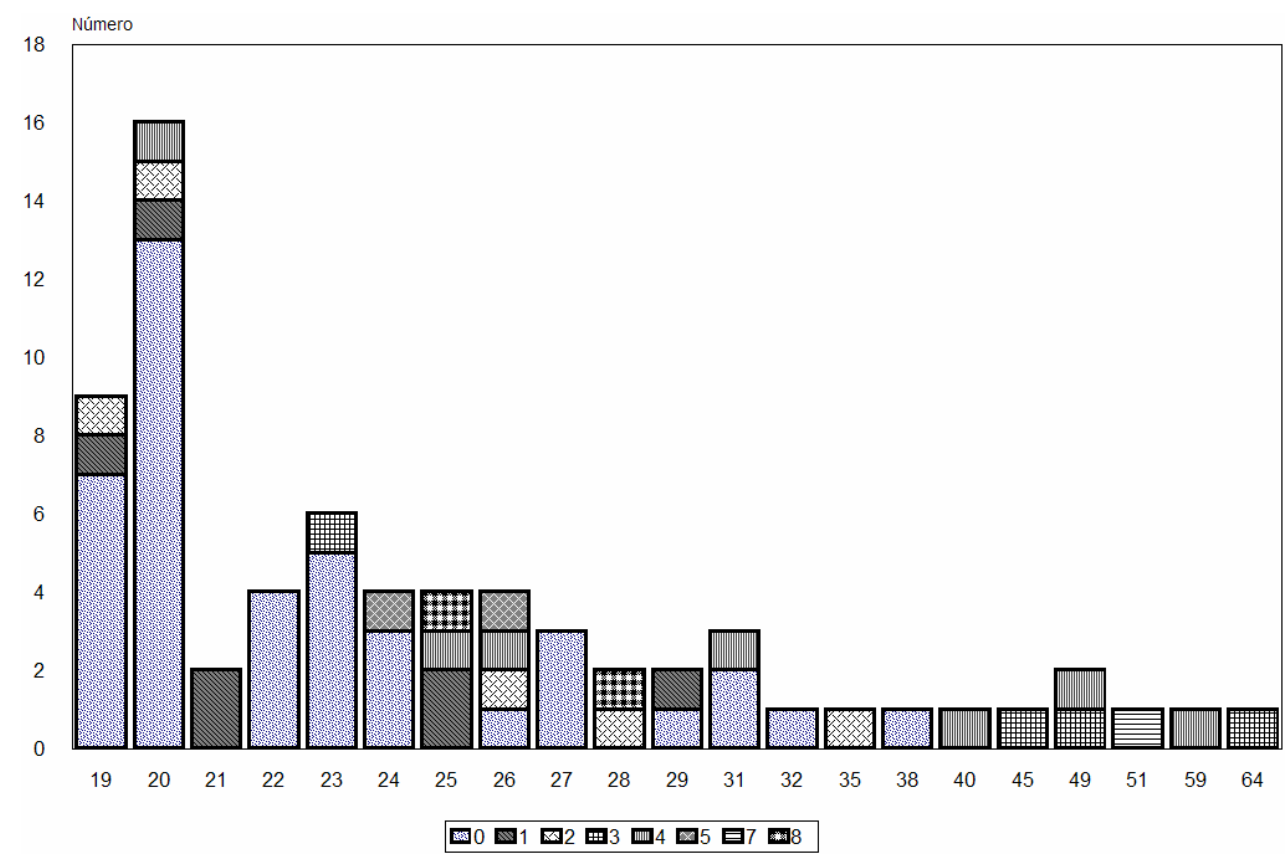

Gráfico 2. Escores (número absoluto e percentual) SALSA e EHF da população do estudo.

de casos multibacilares traduzia a situação epidemiológica do município e do Estado ${ }^{(21,22)}$. Além disso, a maior concentração de casos entre multibacilares permite a análise da relação entre incapacidade física e as dimensões avaliadas pelas escalas.

Nessa perspectiva, de acordo com a validação realizada da escala SALSA ${ }^{(9)}$, seria esperado Que Quanto maior o escore EHF maior o escore SALSA, refletindo Que a limitação funcional leva à limitação de atividades. Neste estudo esta relação foi observada parcialmente, pois alguns participantes tiveram comportamento inesperado. Dois participantes Que tiveram maior pontuação no EHF (8) estavam agrupados nos escores 25 e 28 da SALSA. Apenas um participante teve correlação esperada, tendo pontuado 7 no EHF e 51 no escore SALSA.

Com relação à consciência de risco constatou-se Que existe uma 


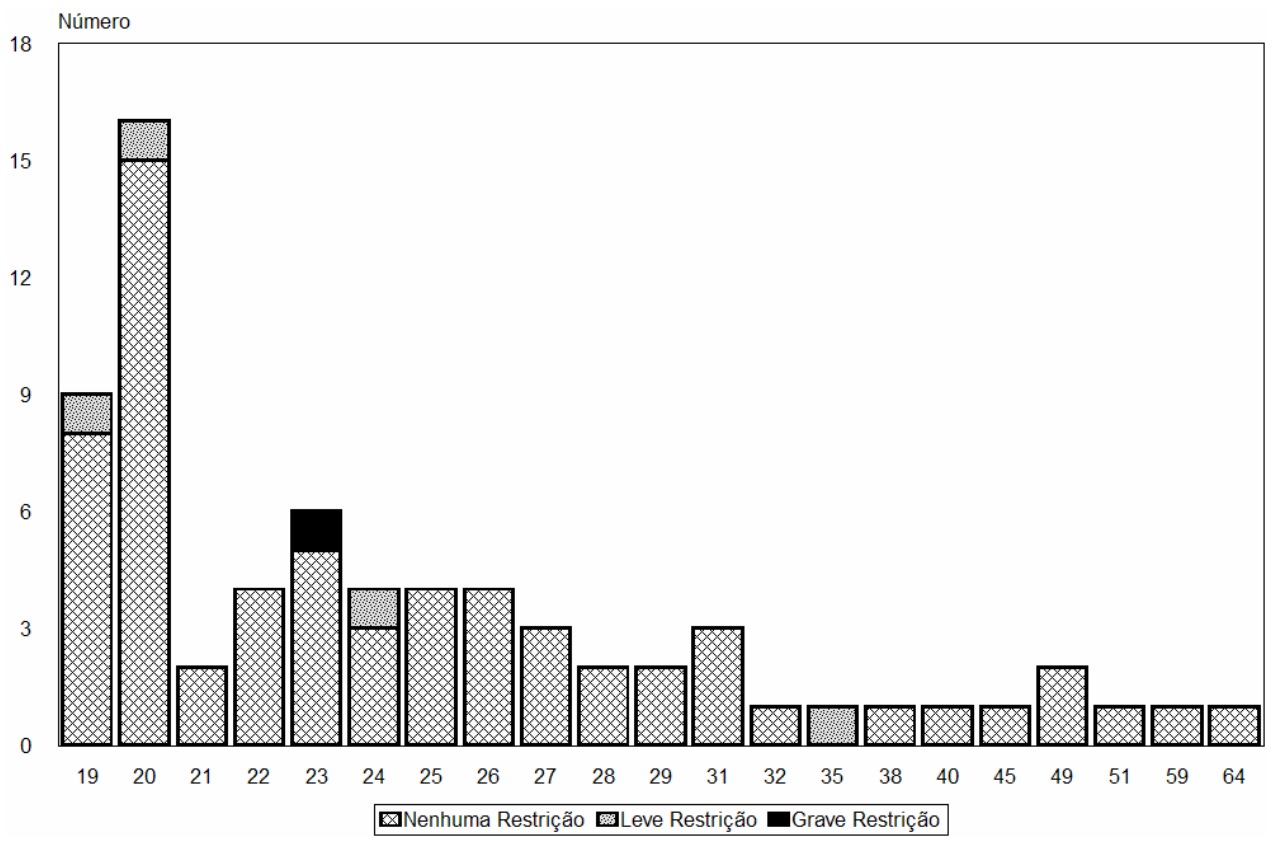

Gráfico 3. Escore (número absoluto e percentual) SALSA e Escala de Participação da população do estudo.

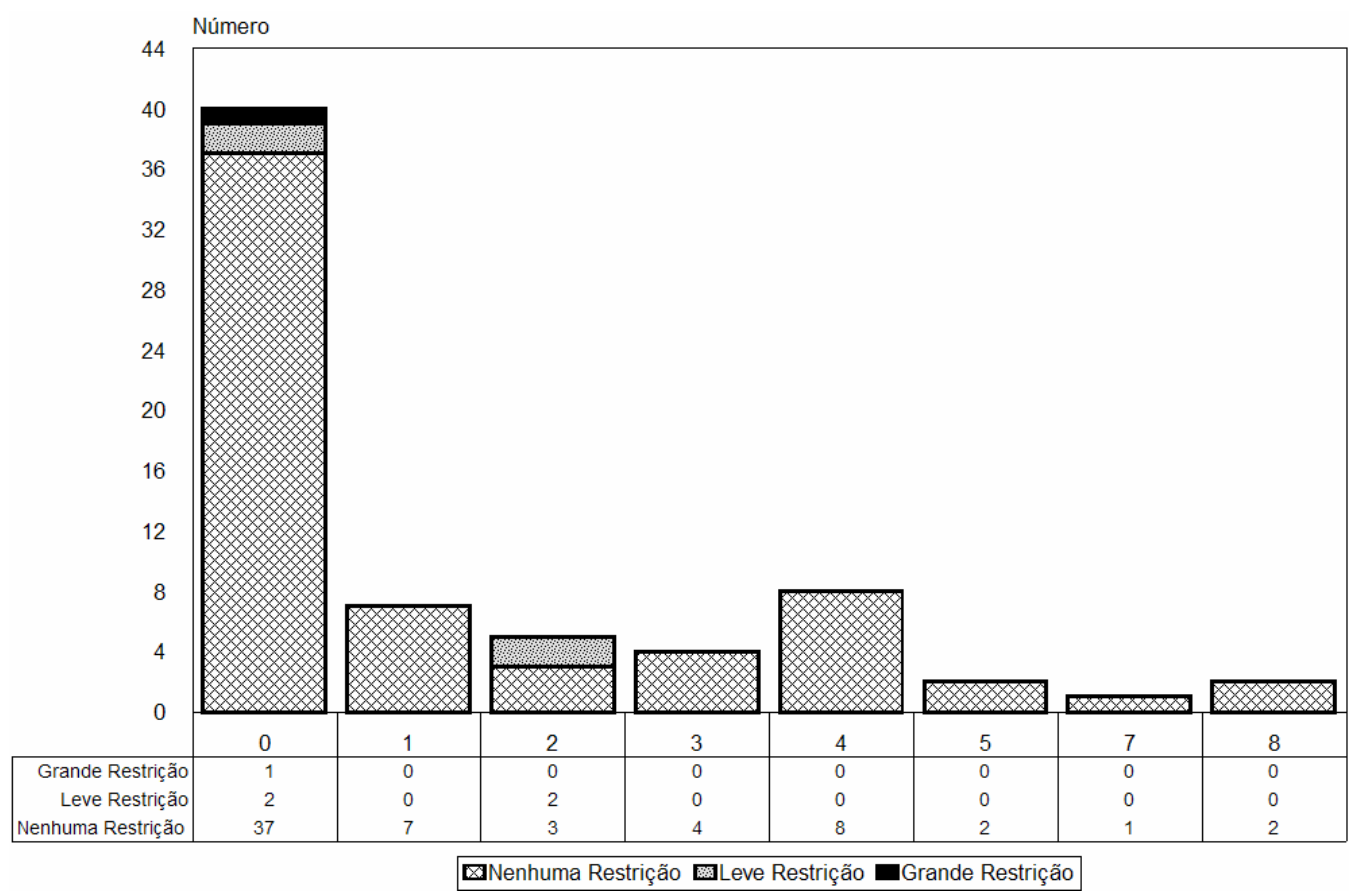

Gráfico 4. Escore (número absoluto e percentual) EHF e Escala de Participação da população do estudo.

baixa percepção de risco dos participantes. Muitas situações observadas junto às pessoas avaliadas foram decorrentes do fato delas não conseguirem fisicamente.

Das 4 (5,8\%) pessoas Que foram classificadas como tendo leve restrição à participação, duas apresentavam dificuldade para encontrar trabalho e adotavam uma postura de reclusão por "medo do marido" e foram caracterizadas como tendo: escore SALSA abaixo de 30 , EHF zero, idade de 42 e 60 anos. A terceira pessoa apresentou alterações físicas, escore SALSA 40, EHF 4 e idade 78 anos. O último tinha idade 36 anos, 1 ano 8 meses de tempo de pós-alta, escore SALSA 51 , EHF 7 e escore final na escala de participação 22 , com leve restrição. A pessoa Que apresentou grande restrição, tinha 58 anos, I ano e 9 meses de pós-alta, escore SALSA 23, EHF zero, teve depressão durante o tratamento e ainda estava necessitando de atendimento psicológico.

Os dados destes casos em particular, revelam Que a aplicação da 
escala de participação, mesmo com a associação da escala SALSA, sem a caracterização do contexto de vida da pessoa entrevistada, não permite a afirmativa de Que a restrição identificada é resultado exclusivo da hanseníase. Essa observação já está inserida no manual de referência da escala de participação: recomenda-se a associação de um estudo Qualitativo para os casos analisadas para enriQuecimento da análise ${ }^{(13)}$.

A escala de participação foi de fácil utilização e poderia ser utilizada principalmente durante o tratamento específico com a PQT, pois representa o período em Que a pessoa enfrenta as maiores dificuldades de aceitação da doença e de aceitação pela sociedade, o Que muitas vezes leva à restrição na participação social. Apesar disto, reconhece-se a necessidade de treinamento básico prévio para entendimento dos objetivos e do processo de aplicação da escala. A Questão do estigma é freqüente em pessoas atingidas pela hanseníase, mas neste estudo não foi identificado como um problema relevante pelas pessoas no momento do pós-alta. No entanto, duas pessoas ao serem entrevistadas relembravam Que no momento da PQT a situação era realmente estigmatizante.

A escala de participação pode ser utilizada para avaliar a necessidade sócio-econômica de reabilitação e para avaliar grupos de pessoas, como parte do planejamento de recursos ou programas $^{(13)}$.

Neste estudo percebeu-se Que a escala também possibilita uma abordagem direcionada para aspectos emocionais facilitando a identificação da necessidade de abordagem individual ou em grupos. A identificação da necessidade do atendimento psicológico não invalida e nem dificulta a utilização desta considerando Que o psicólogo é parte da equipe de saúde.

Ressalta-se o avanço entre a versão 4.1 e 4.6 do manual com a definição de graus de restrição à participação, pois anteriormente todas as pessoas Que alcançaram um escore final de 12 eram consideradas como tendo restrição à participação ${ }^{(13,23)}$.

Em relação à avaliação física, a aplicação do EHF, como definido em outros estudos ${ }^{(18,24,25)}$, apresenta uma maior precisão diagnóstica do Que a avaliação do maior grau de incapacidade, adotada no Brasil ${ }^{(16)}$ e em outros países ${ }^{(5)}$. Essa abordagem possibilitou uma avaliação mais acurada com as outras avaliações realizadas neste estudo, principalmente em relação à escala SALSA.

\section{CONCLUSÕES}

O uso da escala SALSA neste estudo foi de fácil aplicação e pode ser utilizada não apenas em situações de pós-alta, mas também de modo sistemático ao longo do tratamento específico com a PQT. Nesse último caso, a proposta seria de se garantir o planejamento de uma abordagem integral mesmo após a definição de alta por conclusão da PQT. Essa abordagem daria subsídio para identificar também a necessidade da realização de uma avaliação da limitação funcional com maior periodicidade. Outro aspecto válido é a potencialidade de se medir a consciência de risco e de se orientar Quanto o domínio específico a ser abordado, principalmente mãos ou pés.

Este estudo reafirma a potencialidade da aplicação destas ferramentas para a Qualificação da atenção aos portadores de hanseníase com vistas à integralidade da atenção, ultrapassando os limites da abordagem das Questões físicas associadas à doença. Nessa perspectiva, ressalta-se a sua utilidade na avaliação rotineira dos portadores de hanseníase inseridos em programas de prevenção de incapacidades e reabilitação ${ }^{(13)}$.

No cenário do Município de Sobral e do Brasil muitos casos novos ainda são detectados e muitos portadores de hanseníase ainda apresentam grandes necessidade nos campos físico, social, econômico e psicológico. Portanto, a ampliação da capacidade de avaliação tornase um grande desafio. Incorporar a abordagem das limitações de atividades e /ou de restrições à participação representa um possível caminho para o alcance de intervenções eficientes de reabilitação Que direcionem para uma abordagem integral e o seu monitoramento.

\section{REFERÊNCIAS}

1. World Health Organization. Leprosy Situation, 2008. Weekly Epidemiol Record 2007; 83(33): 293-300.

2. Ministério da Saúde (BR). Secretaria de Vigilância à Saúde. Situação Epidemiológica da Hanseníase no Brasil 2001 a 2006. Brasília: Ministério da Saúde; 2007.

3. Ministério da Saúde (BR). Secretaria de Atenção à Saúde. Departamento da Atenção Básica. Inform Atenção Básica 2007; 8(42).

4. Bakirtzief Z. Identificando barreiras para aderência ao tratamento de hanseníase. Cad. Saúde Pública 1996; 12(4): 497-505.

5. International Leprosy Association Technical Forum (ILA). Report of the International Leprosy Association Technical Forum. Int I Lepr Other Mycobact Dis 2002; 70( I-Suppl): S2-S62.

6. Van Brakel, WH, Anderson, AM, Mutatkar, RK, Bakirtzief, Z, Nicholls, PG, Raju, MS, Das-Pattanayak, R.K The Participation Scale: Measuring a key concept in public health. Disabil Rehabil. 2006; 28(4): 193-203.

7. Van Brakel, W.H. Measuring leprosy stigma-a preliminary review of the leprosy literature. Int I Lepr Other Mycobact Dis, 2003; 7I(3): 190-7.
8. Federação Internacional de Associações Anti-Hanseníase (ILEP) Guia para aReabilitação Sócio-Econômica de Pessoas Afetadas pela Hanseníase. Londres: ILEP; 1999.

9. Ebenso I. The development of a short Questionnaire for screening of activity limitation and safety awareness (SALSA) in clients affected by leprosy or diabetes. Disabil Rehabil 2007; 29(9): $689-700$.

10. Tsutsumi A, Izutsu T, Islam A. Maksuda AN, Kato H, Wakai, S The euality of life, mental health, and perceived stigma of leprosy patients in Bangladesh. Soc Sci Med. 2007; (64): 2443-53.

11. Organização Mundial da Saúde. Classificação Internacional de Funcionalidade, Incapacidade e Saúde. São Paulo: EDUSP; 2003.

12. World Health Organization. Global Strategy for Further Reducing the Leprosy burden and sustaining Leprosy control activities 2006-2010, Geneva: WHO; 2005.

13. Participation Scale Users: Manual v.4.6. [cited on 2006 feb 12]. Disponível em: http://www.leprastichting.nl/assets/infolep/ Participation\%20Scale\%20Users\%20Manual\%20v.4.6.pdf

14. Ministério da Saúde (BR). Portaria n ${ }^{\circ}$. 1.838, de 09 de outubro de 2002. Define as seguintes diretrizes e estratégias para o 
cumprimento da meta de eliminação da hanseníase como problema de saúde no Brasil até 2005. Brasília: Ministério da saúde; 2002.

15. Prefeitura Municipal de Sobral. Sobral em dados. Sobral; 2007. [citado em: 22 dez 2007]. Disponível em: http:// www.sobral.ce.gov.br/comunicacao/novo2/index.php? pagina $=$ cidade $/$ sobral-em-dados.php

16. Ministério da Saúde (BR). Secretaria de Políticas de Saúde. Departamento da Atenção Básica. Guia para o controle da hanseníase. Brasília: Ministério da Saúde; 2002.

17. De Rijk AJ, Gabre S, Byass P, Berhanu T. Field evaluation of WHO-MDT of fixed duration at ALERT, Ethiopia: The AMFES project - I. MDT course completion, case-holding and another score for disability grading. Lepr Rev 1994; 65:305-19.

18. Van Brakel WH, Reed NK, Reed DS. Grading impairment in leprosy. Lepr Rev1999; 70(2): 1 80-8.

19. Escala SALSA: pacote de teste Beta - versão 1.0. [citado em: 3 I maio 2005]. Disponível em: http://www.almbrasil.com.br/ arquivos/Escala\%20SALSA.doc
20. Dijkers M. Comments on Van Brakel Participation Scale. Disabil Rehabil 2006; 28(21):1360-2.

21. Ministério da Saúde (BR). Departamento de Informática do SUS - DATASUS. Informação em saúde. Brasília: Ministério da Saúde; 2006.

22. Campos SSL, Ramos Ir AN, Kerr-Pontes LRS, Heukelbach J. Epidemiologia da hanseníase no Município de Sobral, Estado do Ceará-Brasil, no Período de 1997 a 2003. Hansen Int 2005; 30(2): 167-73.

23. Escala de Participação: Manual do usuário - versão 4. l . [citado em: 31 maio 2005]. Disponível em: http://www.almbra sil.com.br/arquivos/4.ManualdoUsuario\%20daBB6D.doc

24. Meima A, Saunderson PR, Gebre S, Desta K, Habbema JDF. Dynamics of impairment during and after treatment: the AMFES cohort. Lepr Rev 200 I ; 72(2): 158-70.

25. Reed Nk, Van Brakel WH, Reed DS. Progresso for Impairment Scores Following Commencement of Chemoherapy in Multibacillary Leprosy Patients. Int I Lepr 1997; 65(3): 328-36. 